\title{
A Study on the Mobile Partial Discharge Locating System
}

\author{
Yan Yang ${ }^{1}$, Lin Tang ${ }^{2}$, Yue $\mathrm{Hu}^{2}$, Qian Wang ${ }^{1}$, Gaolin $\mathrm{Wu}^{1}$, Tianchen Zhang ${ }^{2}$, Xiuchen Jiang ${ }^{2}$ \\ ${ }^{1}$ Electric Power Research Institute of Chongqing, Chongqing, China \\ ${ }^{2}$ Key Laboratory of Control of Power Transmission and Conversion, Ministry of Education, Shanghai Jiao Tong University, \\ Shanghai, China \\ Email: yuehu@sjtu.edu.cn
}

Received April, 2013

\begin{abstract}
This paper studies on the Partial Discharge (PD) Locating System based on a mobile array of ultra-high frequency (UHF) antennas and a vehicle-mounted PD locating system is established. The system consists of omni-directional antenna array for receiving UHF PD signals, a pre-processing circuit for signal amplification and filtering and the highspeed acquisition and control unit of PD pulse signals. The developed locating system is able to simultaneously record the PD pulse signals received by the antenna array. By assessing the time difference of arrival (TDOA), the two-dimensional hyperbolic locating model quickly locates the PD source. Based on the software developed by LabVIEW, it is also possible to display, store and further analyze the acquired signals. Through the simulation of PD signals and the locating experiments with the system, it is proved that the PD locating system possesses the features of rapidity and precision in determining the bearing of PD source.
\end{abstract}

Keywords: Partial Discharge; Locating System; Two-Dimensional Model; Mobile

\section{Introduction}

A substation is the important part of power system. The normal operation of electrical equipments in the substation is the key to the security and reliability of the power system. Therefore, monitoring the operation of the equipments at the substation is of significance. The study shows that PD can be used to detect the insulation defects of high-voltage equipments. In the meantime, most PD facilitates the insulation deterioration of the electrical equipments, ultimately resulting in insulation failure and electric accident [1]. For this reason, the detection and locating of PD is highly important. At present, the main approaches to PD locating include ultrasonic locating method, electrical locating method and UHF electromagnetic wave locating method [2-4], which are primarily designed for the single equipment such as GIS (Gas Insulated Switchgear), transformer and capacitive equipments. During the normal operation, any high-voltage power equipments in the substation are susceptible to PD. It is necessary to install monitoring device on every power equipment if the whole substation is be monitored. In this case, the cost will be extremely high, while the efficiency of the monitoring system is low. Moreover, the maintenance of the devices requires considerable work. It is indicated that the UHF method has high sensitivity and strong disturbance suppression to PD. Recently, there have been studies on the on-line monitoring of
UHF PD in the substation based on the antenna array [4, 5]. A Few reports are on the UHF PD locating based on fixed site antenna array [6]. The sensors are immobile. So there are some blind areas of the detection of PD signals in the whole substation. At the same time, as the sensors are distant away from the high-voltage power equipments, PD signals are attenuated during transmission, resulting in signal-to-noise ratio and signal processing difficulty. As a consequence, the precision locating is restricted. The PD locating method based on the vehicle-mounted array of UHF sensors can avoid these problems very well. By exploiting the mobile feature of the sensor array, the locating result could be verified for several times at different places, which improves the precision and efficiency. This study designs and establishes a set of vehicle-mounted PD locating system. Its rapidity and precision have been verified through experiments, with accurate PD location.

\section{Two-Dimensional Location Algorithm}

\subsection{Bearing Algorithm}

The basic principle of UHF locating method is TDOA. Several sensors are used at different places to receive UHF signals from one PD source. Then TDOA is estimated through energy accumulation or relevant algorithms, based on which the mathematical model is established, including 3-dimensional model, hyperboloid 
model and spherical model. By using the Newton iteration method to solve the nonlinear equations set up based on the locating model, the precise locating of PD source is achieved. The study shows that by using the abovementioned method, the locating precision is largely affected by the estimated TDOA, in a non-linear manner. In order to improve the precision of calculation of TDOA, the sampling frequency of the digital instruments should be very high, leading to high cost of locating system. Moreover, the effect is especially satisfactory when detecting the signals from a single set of equipment within a short distance. However, when PD source is distant away from the UHF sensor, the error of TDOA is still large even with over sampling equipment. The vehicle-mounted on-line monitoring and locating system of PD is characterized by mobility, making it possible to proceed along the route of inspection and stay close to the power equipment to be detected. Based on this feature, this study puts forward the PD locating method using two-dimensional hyperbolic locating model (see Figure 1). Compared with the three-dimensional locating model, this method only determines the bearing of PD source relative to the array of sensors, achieving low precision of TDOA calculation. After using the location algorithm for the power equipments suspected of PD, other portable devices for PD detection could also be used for further detection and precise location. The specific location model is as follows:

In order to obtain the bearing of PD source, only the coordinates of $\mathrm{X}$ axis and $\mathrm{Y}$ axis are required. In the two-dimensional hyperbolic locating model, it is assumed that the power equipment and the UHF sensors are in the same plane. As Figure 1 shows, $\mathrm{P}$ ( $\mathrm{x}, \mathrm{y}$ ) is taken as the position of PD source and the UHF sensor is located at $i$ or $j(i, j=1,2,3,4)$. The distance from $P$, the

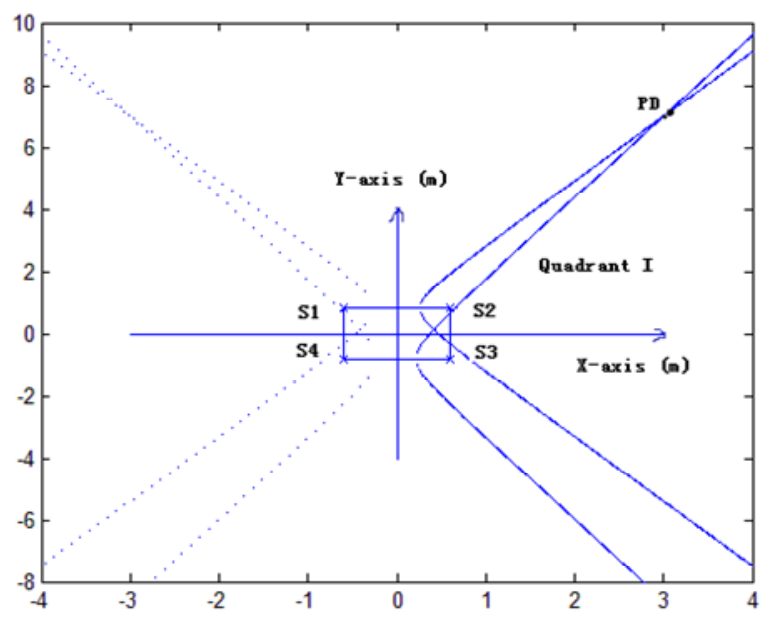

1. Locating algorithm diagram (top view).

Figure 1. Locating algorithm diagram (top view).
PD source, and the sensor is $d_{i}, d_{j}$. In the process of PD detection, TDOA at the UHF sensor receiving the UHF PD signals can be calculated. Assuming the transmission velocity is constant (velocity of light), the difference of distance $d_{i}-d_{j}$ could be obtained. By making one difference constant (for example, $\mathrm{d}_{1}-\mathrm{d}_{2}$ is constant, see Equation (1)), a unique single-branch hyperbolic curve could be determined. According to the TDOA detected by different arrays of sensors (one array consists of any two UHF sensors), different single-branch hyperbolic curves are obtained. Any two single-branch hyperbolic curves, as shown in Figure 1, could form binary quadratic equations as Equation (1). Then the two-dimensional coordinates of PD source could be obtained by analytical solution. By means of coordinate transformation, the bearing and the radial distance could be known. The six independent delta- $T$ calculated from antenna array can determine the quadrant where PD happens. So the other wrong intersections can be eliminated. Such as, if PD source discharges in Quadrant I,dealt-T21(which means T2-T1) must be negative.

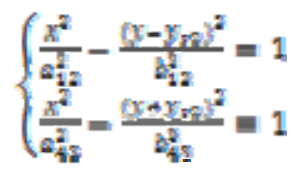

where $a_{i j}=\left(d_{i}-d_{j}\right) / 2$ is length of semi-major axis;

$\mathrm{b}_{\mathrm{ij}}{ }^{2}=\mathrm{c}_{\mathrm{ij}}{ }^{2}-\mathrm{a}_{\mathrm{ij}}{ }^{2}$ is the square of the length of semi-minor axis; $c_{i j}$ is the focal distance of hyperbola, determined by the size of antenna array

\subsection{Arrival Time Difference Calculation}

In the hyperbolic locating model, it can be seen that the detection method of bearing of PD source is directly based on TDOA of UHF PD signals. The error of the calculated TDOA will inevitably affect the precision of location. The System uses the cross-correlation algorithm to estimate TDOA [7]. In the calculation of TDOA, it is assumed that the sampling rate of locating system is $5 \mathrm{Gps} / \mathrm{s}$, which means that there is a deviation of sampling interval. As a result, the error of the estimated TDOA is \pm 0.2 ns. . When the noise level is high, especially when the peaks of PD signal are close, the TDOA calculated by the cross-correlation algorithm will be affected. The PD signal is the pulse signal with short PD duration. When the noise signal of the wave front and wave tail is large, mistaken translation points may occur, leading to maximal correlation coefficient. In order to obtain a relatively precise TDOA, the system adopts the strategy of combining hardware filtering with software de-noising. The hardware filtering is accomplished by the front-end conditioning circuit. The purpose is to enable the sampling system to acquire PD signal during a single collection task. The front-end conditioning circuit 
amplifies the PD signal on a specific basis, while attenuating the nose signals. The de-noising with software is based on self-adaptive wavelet de-noising. Taking the signal without being contaminated by PD signal as background noise, it divides the db8 wavelet into 8 layers. The wavelet threshold is extracted for de-noising of the original signal (Figure 2). After wavelet de-noising for PD signal detected at a substation of Shandong Province, the result is as shown in Figure 3.

Comparing the signals in Figure 2 and Figure 3, it is easy to see that the system well retains the PD pulse signal and removes the background noise. The signal-tonoise ratio stays relatively high

\section{Mobile PD Locating System}

According to the two-dimensional bearing locating model, the vehicle-mounted PD locating system is designed. The composition of the system and the procedures of signal processing are shown in Figure 4.

The system mainly consists of:

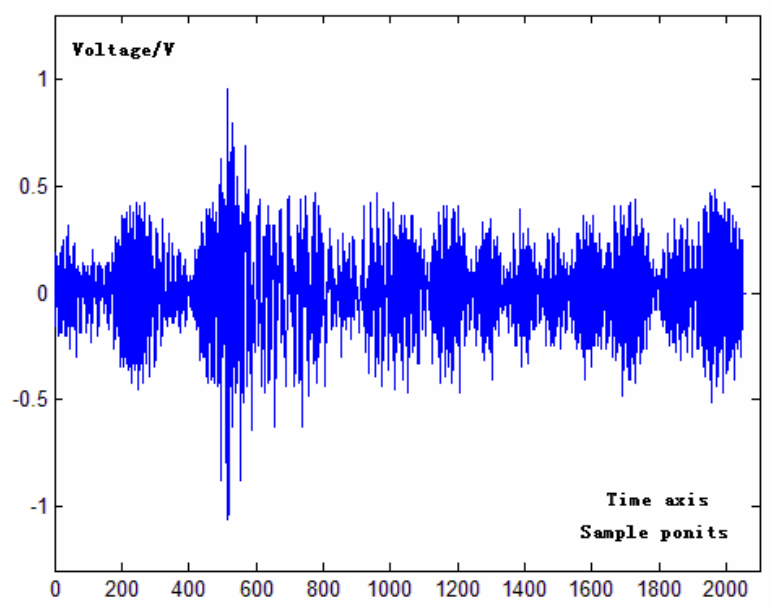

Figure 2. PD Signal Detected in a Substation of Shandong Province.

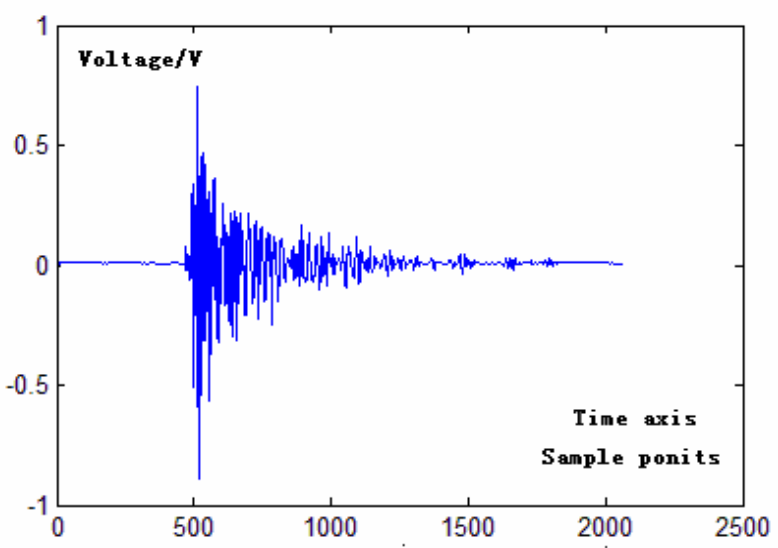

Figure 3. Signal after Wavelet De-noising.

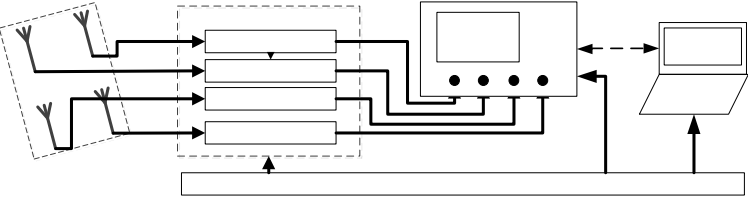

Figure 4. System structure.

1) UHF sensor array: The sensor's response frequency is $0.2-3 \mathrm{GHz}$ in order to ensure that the sensor array could receive all pulse signals emitted by a variety of PD insulation defects, which produces TDOA. Omnidirectional sensor array includes 4 sensors with $1 \mathrm{~m}$ distance between any two. The sensors are arranged in a rectangular plane.

2) The pre-processing circuit: It is composed of the same four-channel conditioning circuit which is mainly for signal amplification and wave filtering. According to the actual noise frequency spectrum of substation, a band-pass filter amplifier is designed with amplifier gain of $40 \mathrm{~dB}$. The pass-band range of the filter is adjustable with bandwidth of more than $100 \mathrm{MHz}$.

3) Data acquisition unit: The experimental system is DP05204 Digital Storage oscilloscope produced by Tektronix. Under four-channel simultaneous sampling, the highest sampling rate is set as $5 \mathrm{Gps} / \mathrm{s}$.

4) Data analysis and control unit: The hardware is portable computer. The software is developed based on LabVIEW. It performs the functions of controlling data acquisition, data analysis and display, PD source locating and interactive interface.

5) Power supply unit: It adopts the vehicle-mounted mobile power. Lithium batteries supply DC power to the pre-processing circuit. At the same time, inverter is used to provide $220 \mathrm{~V}$ AC voltage for oscilloscope. The capacity of lithium battery is $12 \mathrm{~V} 100 \mathrm{AH}$. After being fully charged, the system is assured to run for more than 8 hours inspecting the substation.

The procedures of signal processing based on the hardware component are as follows: sensor array receives the PD signal from the same source. After the amplifying and filtering by pre-processing circuit, data acquisition unit then digitizes the signal by pulse triggering. The recorded UHF PD pulse waveforms of the same source are then transmitted to the data analysis and control unit for wavelet de-noising and TDOA calculation. Then according to the locating model, all calculated values of TDOA are used for the calculation of the bearing and distance of PD source.

\section{Experiment}

The UHF sensor array is installed on roof of the vehicle through a bracket. By using the simulator of PD at spe- 
cific points, the test on the system's performance in locating is carried out, as shown in Figure 5.

The vehicle-mounted antenna array and interactive interface are shown in Figure 6.

The test data and locating results are shown in Table 1.

The results of the test show that the system could precisely estimate the bearing when locating the PD source simulated by PD simulator. The error is small, typically in the range of $4^{\circ}$. The radial distance estimated deviates greatly. So the precision of the locating remains to be improved.

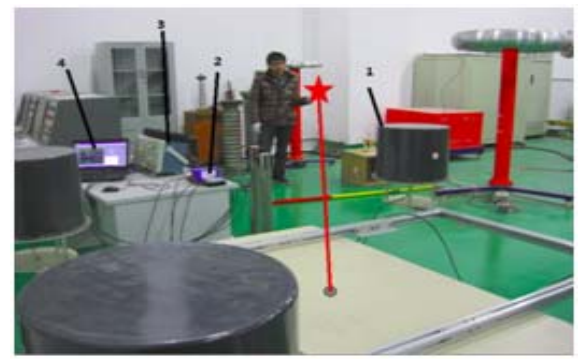

Figure 5. Testing scene. (1-antenna array; 2-amplifier \& filter module; 3-high-speed oscilloscope; 4-laptop).

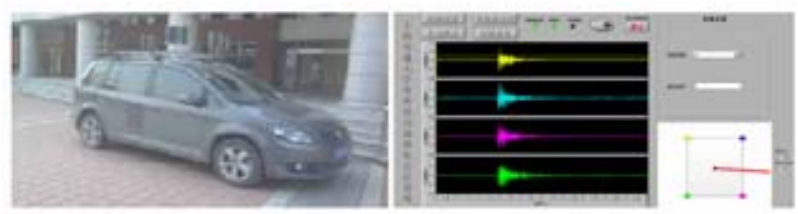

Figure 6. Vehicle-mounted Antenna Array and Interactive Interface.

Table 1. Locating results of the system for PD simulator and the errors.

\begin{tabular}{ccccccc}
\hline & \multicolumn{2}{c}{ Position of PD source } & Locating results & \multicolumn{2}{c}{ Error } \\
\cline { 2 - 7 } & $\begin{array}{c}\text { Bearing } \\
/\end{array}$ & $\begin{array}{c}\text { Distance } \\
/ \mathrm{m}\end{array}$ & Bearing $/{ }^{\circ}$ & $\begin{array}{c}\text { Distance } \\
/ \mathrm{m}\end{array}$ & $\begin{array}{c}\text { Dis- } \\
\text { tance } / \mathrm{m}\end{array}$ & $\begin{array}{c}\text { Distance } \\
/ \mathrm{m}\end{array}$ \\
\hline 1 & 122 & 6.72 & 120.85 & 6.39 & -1.15 & -4.91 \\
2 & 106 & 4.90 & 104.54 & 3.53 & -1.46 & -27.96 \\
3 & 40 & 4.25 & 38.48 & 4.48 & -1.52 & +5.41 \\
4 & -4 & 5.13 & -3.76 & 1.1 & 0.24 & -78.56 \\
5 & 315.00 & 14.14 & 315.17 & 12.26 & 0.17 & -13.30 \\
6 & 326.31 & 18.03 & 327.07 & 35.71 & 0.76 & +98.06 \\
7 & 330.43 & 22.36 & 326.59 & 35.9 & -3.84 & +60.55 \\
\hline & 337.38 & 26.00 & 333.64 & 33.45 & -3.74 & +28.65 \\
\hline
\end{tabular}

\section{Discussion and Conclusions}

This paper designs a vehicle-mounted PD locating system for substation based on the UHF method. The hardware unit is built, consisting of UHF sensor array, filtering and amplifying module, high-speed digital storage oscilloscope, portable computer, LABVIEW interactive interface and the connecting components between parts. In the test where PD simulator is used to simulate PD signal, this system gives a precise locating of PD source. The reliability and the accuracy of the system in determining the bearing of PD source are verified. It possesses a bright future in the application of PD location.

The two-dimensional hyperbolic locating algorithm used by the locating system has superiorities in locating speed and accuracy of bearing determination. However, it still needs to improve the precision of radial distance determination. In addition, further analysis of PD signal which has been collected is needed to realize the classification and pattern recognition of PD

\section{REFERENCES}

[1] S. S. Zhang, J. X. Chen, C .R. Li and H. Lin, "Influence of Windings on Locating Partial Discharge in Transformers by Using Ultra-high Frequency Method,” High Voltage Engineering, Vol. 39, No. 2, 2013, pp. 324-326.

[2] D. J. Li, J. Z. Liang, K. W. Bu, J. G. Yang and Y. M. Li, "Ultrasonic Detection of Partial Discharge on Typical Defects in GIS,” High Voltage Apparatus, Vol. 45, No. 1 , 2009, pp. 72-74.

[3] J. F. Gui, W. S. Gao and Z. S. Wu, "Frequency Band of Pulse Current Method for the Measurment of PD in Transformer,” High Voltage Engineering, Vol. 31, No. 1, 2005, pp. 45-46.

[4] H .J. Hou, G. H. Sheng, P. Q. Miao, X. W. Li, Y. Hu and X. C. Jiang, "Partial Discharge Location Based on Radio Frequency Antenna Array in Substation," High Voltage Engineering, Vol. 38, No. 6, 2012, pp. 1334-1340.

[5] J. M. Philip, I. E. Portugues and I. A. Glover, "Radiometric Location of Partial Discharge Sources on Energized High-Voltage Plant," IEEE Transactions on Power Delivery, Vol. 20, NO. 3, 2005, pp. 2264-2272. doi:10.1109/TPWRD.2004.843397

[6] E. P. Iliana and J. M. Philip, "RF-Based Partial Discharge Early Warning System for Air-Insulated Substation," IEEE Transactions on Power Delivery, Vol. 24, NO. 1, 2009, pp. 20-29. doi:10.1109/TPWRD.2008.2005464

[7] J. Tang and J. Chen, "Time Difference Algorithm Based on Energy Relevant Search in PD Location of Power Transformer," Master Thesis, College of Electrical Engineering of Chongqing University, Chongqing, 2009 